\title{
Transient inactivation of the infralimbic cortex induces antidepressant-like effects in the rat
}

\author{
David A Slattery ${ }^{1,2}$, Inga D Neumann ${ }^{2}$ and John F Cryan ${ }^{1,3}$
}

\author{
Journal of Psychopharmacology \\ 25(10) 1295-1303 \\ (C) The Author(s) 2011 \\ Reprints and permissions: \\ sagepub.co.uk/journalsPermissions.nav \\ DOI: $10.1177 / 0269881110368873$ \\ jop.sagepub.com
}

(3)SAGE

\begin{abstract}
Affective disorders are among the main causes of disability worldwide, yet the underlying pathophysiology remains poorly understood. Recently, landmark neuroimaging studies have shown increased metabolic activity in Brodmann Area 25 (BA25) in depressed patients. Moreover, functional inactivation of this region using deep brain stimulation alleviated depressive symptoms in severely depressed patients. Thus, we examined the effect of a similar manipulation, pharmacological inactivation of the infralimbic cortex, the rodent correlate of BA25, in an animal model of antidepressant activity: the modified rat forced swim test. Transient inactivation of the infralimbic cortex using muscimol reduced immobility, an antidepressant-like effect in the test. Importantly, this activity was not the result of a general increase in locomotor activity. Activation of the infralimbic cortex using bicuculline did not alter behaviour. Finally, we examined the effect of muscimol in animals bred for high anxiety-related behaviour, which also display elevated depression-related behaviour. Transient inactivation of the infralimbic cortex decreased the high inborn depression-like behaviour of these rats. These results show that it is possible to replicate findings from a clinical trial in a rodent model. Further, they support the use of the forced swim test to gain greater understanding of the neurocircuitry involved in depression and antidepressant-action.
\end{abstract}

Keywords

Cingulate, depression, forced swim test, inactivation, neuroimaging, trait anxiety

\section{Introduction}

Affective disorders are amongst the main causes of disability worldwide. Recent advances in neuroimaging technology have enabled insights into the neurocircuitry underlying depression. These studies have shown that a number of cortical and limbic regions have alterations in both volume and metabolism, such as the hippocampus, amygdala, anterior cingulate cortex and caudate nucleus (Bremner, 2002; Davidson et al., 2002; Dougherty et al., 2003; Drevets, 2001; Mayberg et al., 1999; Seminowicz et al., 2004; Sheline et al., 2002). A number of studies have demonstrated that activity of the subgenual cingulate cortex (Brodmann Area [BA] 25) is decreased by antidepressant administration, electroconvulsive therapy and ablative surgery (Goldapple et al., 2004; Mayberg et al., 1999; Seminowicz et al., 2004). Further, deep brain stimulation (DBS) of BA25, which normalized the metabolic hyperfunctioning observed in this region in treatment-resistant depressed patients, resulted in an antidepressant effect (Mayberg et al., 2005). DBS is believed to mediate these effects by activating inhibitory GABAergic afferents in BA25, which in turn results in altered metabolism in downstream limbic sites (Mayberg et al., 2005).

One of the major predisposing factors to major depression is exposure to stress, and the disease is often viewed as a manifestation of an inability to cope with stress (Anisman and Zacharko, 1990; Cryan and Holmes, 2005;
Kessler, 1997; Sullivan et al., 2000). Thus many models and tests for assessing depression-related behaviour in rodents involve exposure to stressful situations. Of these, the forced swim test (FST) is probably the most widely and frequently used, in which antidepressant administration increases active escape-directed behaviours (Cryan et al., 2002, 2005; Petit-Demouliere et al., 2005; Porsolt et al., 1977). Research efforts have also attempted to utilize differences in stresscoping behaviour to generate selectively bred animal lines, which differ based on a specific endophenotype (Cryan and Slattery, 2007). One of the most established of such

\footnotetext{
${ }^{1}$ Neuroscience Research, Novartis Institutes for BioMedical Research, Novartis Pharma AG, Basel, Switzerland.

${ }^{2}$ Department of Behavioural and Molecular Neuroendocrinology, University of Regensburg, Regensburg, Germany.

${ }^{3}$ Department of Pharmacology and Therapeutics, School of Pharmacy, University of Cork, Cork, Ireland.

\section{Corresponding authors:}

David A Slattery, Department of Behavioural and Molecular Neuroendocrinology, University of Regensburg, Regensburg, Germany Email: david.slattery@biologie.uni-regensburg.de

John F Cryan, PhD, Department of Pharmacology and Therapeutics, School of Pharmacy, University of Cork, Cork, Ireland

Email: j.cryan@ucc.ie
} 
models is high anxiety-related behaviour (HAB) and low anxiety-related behaviour (LAB) rats, which are Wistar rats that have been selectively bred for anxiety-related behaviour on the elevated plus-maze (EPM; Landgraf et al., 2007). These breeding lines also have distinct differences in stresscoping style (Keck et al., 2003) and HAB rats have been shown to display a depressive-like phenotype in the FST under basal conditions compared with both LAB and Wistar rats (Keck et al., 2003; Slattery and Neumann, 2010). Therefore, HAB rats represent an established animal model to study the underlying aetiology of affective disorders and may more closely mimic the situation observed in patients suffering from depression.

The rodent correlate of the human BA25 is believed to be the infralimbic cortex (Barbas et al., 2003; Ongur and Price, 2000; Quirk and Beer, 2006; Uylings et al., 2003), which forms part of the prefrontal cortex (PFC) together with the prelimbic and anterior cingulate cortices. The infralimbic cortex projects to numerous regions involved in the emotional and neuroendocrine control of stress responses (Ongur and Price, 2000), and a number of studies have linked the infralimbic cortex with stress regulation. A recent study in rhesus monkeys found a positive correlation between subgenual PFC activity and increasing plasma corticosterone levels across a range of stimuli (Jahn et al., 2009). Amat et al. (2005) demonstrated that, together with the prelimbic cortex, the infralimbic cortex in rats inhibits stress-induced activation of the dorsal raphé nucleus when a stressor is controllable; when an operant response (wheel turn) enables escape from a footshock. Furthermore, both acute $(10 \mathrm{~min}$ exposure to swim stress in mice) and chronic (restraint in rats) stress result in dendritic retraction in the infralimbic cortex (Goldwater et al., 2009; Izquierdo et al., 2006; Perez-Cruz et al., 2009). This retraction was shown to be behaviourally relevant as inhibition of protein kinase $\mathrm{C}$ signalling in the PFC prevented both the retraction and cognitive deficits induced by chronic stress (Hains et al., 2009). Additionally, chronic stress-induced PFC reorganization has been shown to result in a shift to habitual strategies and a lack of behavioural flexibility (Dias-Ferreira et al., 2009).

Taken together, these results, combined with the human DBS study, suggest that the infralimbic cortex may be an important locus for the modulation of depression-related behaviours. Therefore, the aim of the present study was to determine whether inactivation of the infralimbic cortex would alter depression-like behaviour in the rat FST in an analogous manner to that shown in humans. In order to do so we used the $\mathrm{GABA}_{\mathrm{A}}$ receptor agonist (muscimol) to inactivate, and the $\mathrm{GABA}_{\mathrm{A}}$ receptor antagonist (bicuculline) to activate the infralimbic cortex. $\mathrm{GABA}_{\mathrm{A}}$ receptor ligands were chosen for a number of reasons: (1) it is hypothesized that GABAergic effects within BA25 are mediating the actions of DBS to lower metabolic activity in this region and induce an antidepressant effect in patients (Mayberg et al., 2005); (2) the use of ligands to activate and inactivate the same neurobiological system enables direct comparison of these opposing states; (3) localization of $\mathrm{GABA}_{\mathrm{A}}$ receptor subunits have been extensively described in the PFC; (4) there is an expansive literature using muscimol to inactivate various brain regions (Corcoran et al., 2005; Maren et al., 2001; Pothuizen et al., 2005; Zhang et al., 2002).
Thus, we determined whether inactivation of the infralimbic cortex could modulate depression-related behaviour in normal laboratory animals. Our second aim was to determine whether it could reduce high inborn levels of depressionrelated behaviour in $\mathrm{HAB}$ rats in an analogous fashion to that observed following DBS in treatment-resistant patients. Thus, these experiments were designed to provide insight into whether it is feasible to take a direct finding from a human study and verify it in animal models of stress-induced coping behaviour.

\section{Materials and methods}

\section{Animals}

Male Sprague-Dawley rats (Charles River, France) or male $\mathrm{HAB}$ rats (from our breeding colony at the University of Regensburg) (Landgraf and Wigger, 2002; Neumann, 2009) weighing between 270 and $300 \mathrm{~g}$ at the time of surgery were used in these studies. The animals were housed in groups of 2-4 and maintained on a 12-h light:dark cycle (lights on 06:00) in a temperature controlled colony $\left(22-24^{\circ} \mathrm{C}\right)$. The animals had free access to food and water. Animals were allowed to habituate for at least 7 days before surgery. After surgery all animals were singly housed. All experimental procedures were subject to institutional review and conducted in accordance with the Veterinary Authority of Basel-Stadt, Switzerland and the local government of the Oberpfalz.

\section{Activation/inactivation experiments}

These experiments were carried out as previously described for the anterior cingulate cortex (Bissiere et al., 2006). In order to activate or inactivate the infralimbic cortex, bilateral infusion of, respectively, the $\mathrm{GABA}_{\mathrm{A}}$ receptor agonist muscimol (200 pmoles per hemisphere, Sigma Aldrich Biochemicals) or the $\mathrm{GABA}_{\mathrm{A}}$ receptor antagonist bicuculline (50 pmoles per hemisphere, Sigma Aldrich Biochemicals) were performed prior to the behavioural task via bilateral guide cannulae. Drug concentrations and the time-frame from point of infusion were chosen from previous intracranial studies (Bissiere et al., 2006; Dickinson-Anson and McGaugh, 1997; McDougall et al., 2004).

\section{Drug infusion}

The cannulae (28 gauge) used for infusion into the infralimbic cortex were cut $4.5 \mathrm{~mm}$ longer than the guide cannula (i.e. $12.5 \mathrm{~mm}$ ) and were connected to $10-\mu 1$ Hamilton syringes (Hamilton, USA) placed inside a syringe driver (CMA, Sweden) via $0.28 \mathrm{~mm}$ ID polyethelene tubing. The needles were carefully inserted into the guide cannula and drugs were simultaneously infused into each hemisphere in a final volume of $1 \mu \mathrm{l}$ over $1 \mathrm{~min}$. The cannulae were left in place for $1 \mathrm{~min}$ following the infusion in order to allow diffusion of the drug.

\section{Cannula implantation}

Bilateral guide cannula implantation was performed under isoflurane anaesthesia $(1.5-3 \%$ isoflurane, oxygen 
flow rate $450-500 \mathrm{ccm}$ ). Pre-operative analgesia (buprenorphine $0.1 \mathrm{mg} / \mathrm{kg}$; s.c.) was administered $30 \mathrm{~min}$ before surgery. After induction of anaesthesia the rat was placed into a stereotaxic frame with the incisor bar set to $+5 \mathrm{~mm}$ and implanted with bilateral guide cannulae to the surface of the dura ( $8 \mathrm{~mm}$ in length) located above the infralimbic cortex (AP $+4.5 \mathrm{~mm}$ from bregma; $\mathrm{ML} \pm 0.75 \mathrm{~mm}$; V $4.5 \mathrm{~mm}$ ). The cannulae were fixed with two stainless steel skull screws using dental acrylic. The wound was then sutured and animals returned to their home cage and allowed to recover for 7 or 8 days before the experiment began. A dummy cannula $(8 \mathrm{~mm})$ was inserted inside the guide cannula and removed daily and reinserted to ensure that the cannulae remained viable. Additionally, this reduced stress associated with the infusion on the behavioural testing days.

\section{Modified forced swim test}

Sprague-Dawley. The FST was conducted using an adapted protocol from that described previously (Cryan and Lucki, 2000a; 2000b; Cryan et al., 2005; Slattery et al., 2005). Briefly, rats were individually placed into a plexiglass cylinder $(21 \mathrm{~cm} \times 46 \mathrm{~cm})$ filled with $25^{\circ} \mathrm{C}$ water to a depth of $30 \mathrm{~cm}$. The rats were removed after $15 \mathrm{~min}$, dried, and returned to their home cage. Water was changed between each test. Twenty-four hours after this first exposure, the rats were replaced in the swim cylinder (under the same conditions) for $5 \mathrm{~min}$. Both sessions were recorded using a video camera placed above the cylinder for subsequent analysis. Rats were simultaneously infused bilaterally $10 \mathrm{~min}$ prior to each swim session. The rater of the sessions was blind to the treatment group being scored. Using a time sampling technique, the predominant behaviours, climbing, swimming or immobility, in each 5-s period of the first $300 \mathrm{~s}$ of the pre-test swim test and the full $300 \mathrm{~s}$ of the swim exposure were recorded (providing an overall total of 60 scores). Climbing behaviour consisted of upward-directed movement of the forepaws usually along the side of the swim cylinder. Swimming behaviour consisted of horizontal movement throughout the swim chamber, which also included crossing into another quadrant. Immobility was defined as the animal floating in water without struggling and only making movements necessary to maintain its head above the water (see Cryan et al., 2002 for pictorial representations of observed behaviours).

In the traditional rat FST the initial exposure to the test apparatus (often referred to as the pre-test) has been reported to reliably induce immobility upon re-exposure (Borsini et al., 1989). Although this pre-test swim is thought not to be as essential in the modified FST (see Cryan et al., 2005) it has been used systematically to enhance reliability. Therefore, it was important to see whether the infralimbic cortex had a selective role in modulating the initial behavioural responses to the stressful situation, which could potentially affect interpretation of an altered behavioural response in the subsequent test session. This necessitated the infusion of drugs prior to both the pre-swim and the swim test, thus the inclusion of the groups muscimol/vehicle and bicuculline/vehicle. Furthermore, given the role of the infralimbic cortex in working and spatial memory (see Discussion), infusion of these drugs prior to the pre-test swim served as specificity controls for antidepressant-like behaviour.

In this manner, and following histological verification (see below), the effect of vehicle infusion $(n=38)$, muscimol $(n=22)$ or bicucculine $(n=21)$ on pre-test swim behaviour was assessed. These animals were then re-exposed the following day to the swim test and given a second infusion, giving the following groups: vehicle/vehicle $(n=14)$, vehicle/muscimol $(n=13)$, vehicle/bicuculline $(n=11)$, muscimol/vehicle $(n=10), \quad$ muscimol/muscimol $(n=12), \quad$ bicuculline/vehicle $(n=10)$ or bicuculline/bicuculline $(n=11)$.

$H A B$ rats. The modified FST was performed in $\mathrm{HAB}$ rats as described above with the exception of the size of the cylinder $(29 \mathrm{~cm} \times 50 \mathrm{~cm})$. As muscimol and bicucculine infusions were found not to alter behaviour during the pre-swim test in the previous experiment performed in Sprague-Dawley rats, all animals were injected with vehicle $(n=18)$ before the pre-swim and vehicle $(n=8)$ or muscimol $(n=10)$ prior to the swim test.

\section{Effect of infusions on locomotor activity}

Once the data from the FST experiments were available, it was important to assess whether any of the effects of muscimol could be ascribed to a general increase in locomotor activity. In a separate group of Sprague-Dawley rats, implanted with guide cannulae and infused as described above, the effect of drug infusion on locomotor activity was assessed during a 60-min trial in a novel environment, as previously described (Slattery et al., 2005). Briefly, $10 \mathrm{~min}$ after drug infusion rats were placed in the centre of an enclosure $(60 \mathrm{~cm} \times 40 \mathrm{~cm} \times 50 \mathrm{~cm})$ whereby a cage $(55 \mathrm{~cm} \times$ $33 \mathrm{~cm} \times 19 \mathrm{~cm}$ ) was positioned in the enclosure. An activity monitor, consisting of a black and white video camera, was mounted in the top centre of the enclosure and each second a video frame was acquired and assessed with in-house developed software to calculate distance travelled. The animals received bilateral infralimbic infusions of vehicle, muscimol (200 pmoles per hemisphere) or bicuculline (50 pmoles) $10 \mathrm{~min}$ prior to activity testing. Twenty-four hours later, infusions were performed again $10 \mathrm{~min}$ before assessing locomotor activity. In this manner, following histological verification, the following groups were assessed: day 1 , vehicle $(n=18)$, muscimol $(n=8)$, bicuculline $(n=8)$; day 2 , vehicle/vehicle $(n=9)$, vehicle/muscimol $(n=9)$ or bicuculline/bicuculline $(n=8)$.

\section{Histological verification of cannula placements}

Following behavioural testing rats were killed and approximately $1 \mu \mathrm{l}$ of haematoxylin dye was infused into each cannula to assist verification of the infusion sites. The brain was rapidly dissected and snap frozen in isopentane cooled on dry ice. Brains were then stored at $-80^{\circ} \mathrm{C}$ until used for histological verification. Brain sections of $30 \mu \mathrm{m}$ were cryostat-cut and visual verification of the cannula placement was performed with the aid of a brain atlas (Paxinos and Watson, 1986). Only rats with correct bilateral cannulae were used in the statistical and behavioural analyses. 


\section{Data analysis}

For the analysis of the pre-test swim exposure a one-way analysis of variance (ANOVA) was performed. Two-way ANOVA was carried out on all variables in the swim exposure (factors: Day 1 Drug and Day 2 Drug). Repeated measures (RM) ANOVA were performed for the effect of infusion on locomotor activity. For the HAB study, Student's $t$-tests were performed on all behaviours assessed during the swim session. Any overall statistical differences, which were set at $p<0.05$, were further analysed using Fisher's LSD post hoc where appropriate.

\section{Cannula placement}

Nine animals were removed from the FST experiments for incorrect cannula placement (vehicle/vehicle $=1$; vehicle/ bicuculline $=1 ;$ muscimol $/$ vehicle $=3$; bicuculline $/$ vehicle $=2$; bicuculline $/$ bicuculline $=1 ;$ muscimol $/$ muscimol =1), while four animals were removed from the locomotor activity experiments for incorrect cannula placement (vehicle/ muscimol $=1 ;$ muscimol $/$ vehicle $=2$; vehicle $/$ vehicle $=1$ ). No animals were removed from the HAB study.

\section{Effect of infralimbic infusions of muscimol or bicuculline on pre-test swim exposure-induced behavioural changes}

Bilateral infusion of muscimol (200 pmoles per hemisphere) or bicuculline (50 pmoles per hemisphere) into the infralimbic cortex $10 \mathrm{~min}$ before the pre-test swim exposure did not alter any behaviour compared with the vehicle infusion group, that is, immobility time $(F[2,80]=1.23 ; p=0.30)$, swim duration $(F[2,80]=1.85 ; p=0.16)$ and climbing behaviour $(F[2,80]=1.95 ; p=0.15)$. There were no significant differences in pre-test swim activity within each of the subsequent treatment groups on Day 2 (when taking into account rats which received different treatments prior to the swim exposure, that is, between vehicle/vehicle, vehicle/muscimol or vehicle/bicuculline groups in the pre-test swim.

\section{Effect of infralimbic infusions of muscimol or bicuculline on swim exposure-induced behavioural changes}

ANOVA revealed effects of the bilateral infusion 10 min prior to the swim exposure on immobility time $(F[2,80]=5.66 ; p=0.005)$ and climbing behaviour $(F[2,80]=3.30 ; p=0.042)$ but not swimming behaviour $(F[2,80]=0.32 ; p=0.73)$ (for clarity, the results of muscimol and bicuculline groups are represented on separate graphs in Figure 1 despite the statistical analyses being performed on all groups simultaneously). Further analyses revealed a trend for an interaction between pre-test swim $\times$ swim infusion on the duration of swimming $(F[2,80]=3.099 ; p=0.051)$. Posthoc analyses revealed that vehicle/muscimol and muscimol/ muscimol infusion decreased immobility time $(p<0.01)$ while increasing climbing duration $(p<0.05)$ compared with vehicle/ vehicle infusion animals (Figure 1B).

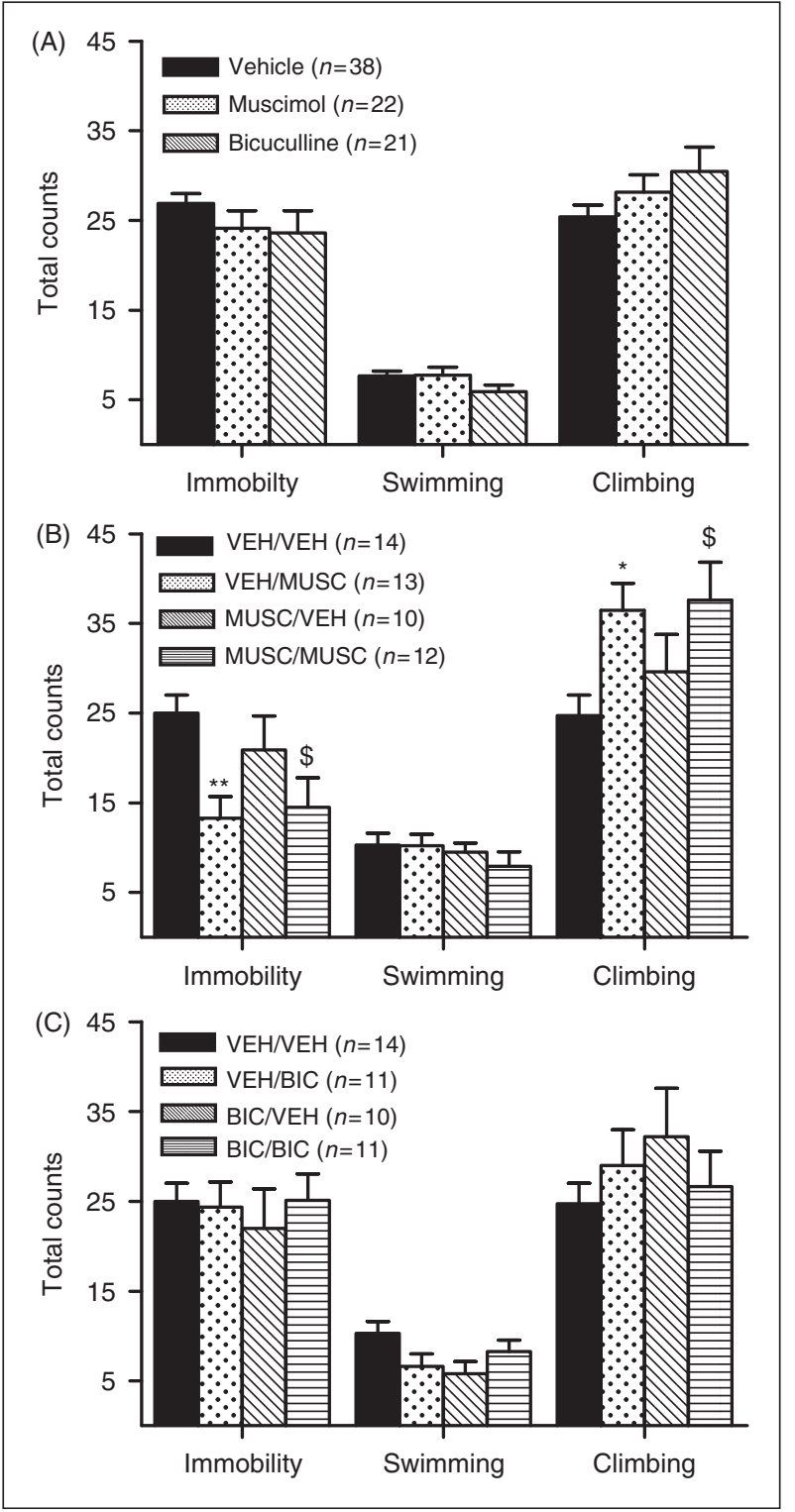

Figure 1. The effect of inactivation/activation of the infralimbic cortex on behaviour in the pre-test $(A)$ and test swim $(B, C)$ exposures. (A) Muscimol (MUSC; 200 pmoles per hemisphere) or bicuculline (BIC; 50 pmoles per hemisphere) $10 \mathrm{~min}$ before the test did not alter any behaviour analysed during the first 5 min of the pre-test swim exposure compared with vehicle (VEH). (B) Muscimol infusion $10 \mathrm{~min}$ prior to the swim exposure on day 2 resulted in a decrease in immobility time mediated via an increase in climbing behaviour compared with vehicle infusion. (C) Infusion of bicuculline prior to the pre-test swim only resulted in a decrease in swimming behaviour during the swim exposure compared with vehicle infusion. Data represent mean \pm SEM of animals with verified bilateral cannula sites. ANOVA was performed followed by Fisher's LSD

post-hoc analyses ${ }^{*} p<0.05 ; \quad{ }^{*} p<0.01$ compared with relevant pre-test swim group; ${ }^{\$} p<0.05$ compared with pre-test swim and swim group. 


\section{Effect of infralimbic infusion of muscimol on swim behaviour in $H A B$ rats}

Student's $t$-test revealed that bilateral muscimol infusion 10 min prior to the swim exposure decreased immobility behaviour $(p<0.05)$ and increased climbing behaviour $(p<0.05)$ but did not affect swimming behaviour $(p>0.05)$ (Figure 2).

\section{Effect of infralimbic infusion of muscimol or bicuculline on locomotor activity}

In order to determine whether the antidepressant-like effects of muscimol may be the result of a general increase in activity, locomotor activity was assessed following the same infusion protocol. Further, the potential for hangover effects of muscimol on locomotor activity was assessed by injecting a group of animals with muscimol prior to the initial test and with vehicle prior to the second test session. Given that bicuculline treatments failed to alter FST behaviour, just one group of animals was used in locomotor studies whereby bicuculline was administered both before the first and second test sessions.

ANOVA revealed no significant effects of infusion $(F[2,28]=0.13 ; \quad p=0.88)$ or infusion $\times$ time interaction $(F[10,240]=0.74 ; p=0.69)$ during the first locomotor activity exposure (Figure $3 \mathrm{~A}$ ). Separate analysis revealed that there were also no statistical differences between the two subsets of the vehicle infused group when taking into account the second infusion (data not shown).

Interestingly, during the second exposure to the locomotor chamber $24 \mathrm{~h}$ later, a significant effect of infusion group $(F[3,30]=6.56 ; p=0.002)$ and a significant infusion group$\times$ time interaction $(F[3,30]=4.53 ; p=0.01)$ was observed. Post-hoc analyses revealed that bicuculline infusion resulted in a pronounced hyperactivity, which lasted for the initial $30 \mathrm{~min}$ of the locomotor activity testing (Figure 3B).

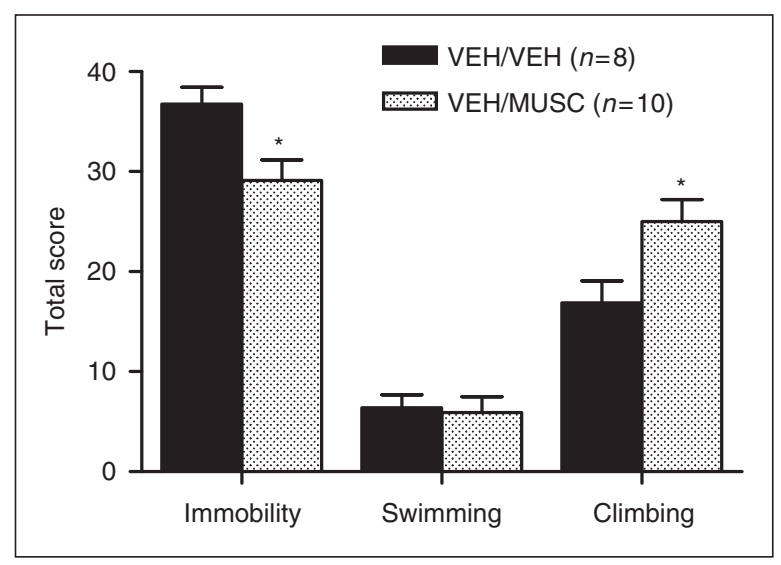

Figure 2. The effect of inactivation of the infralimbic cortex on $H A B$ rat behaviour in the swim exposure. Muscimol (MUSC; 200 pmoles per hemisphere) prior to the swim exposure resulted in a decrease in immobility time mediated via an increase in climbing behaviour compared with vehicle infusion. Student's $t$-tests for each behaviour were performed ${ }^{*} p<0.05$ compared with VEH/VEH treatment.

\section{Discussion}

The recent landmark finding that inactivation of BA25 using DBS could alleviate depressive symptoms in treatment-resistant patients (Mayberg et al., 2005) gave rise to the possibility of examining the effects of a similar treatment in an animal model of antidepressant-activity. Here we demonstrate in Sprague-Dawley rats that, analogous to the clinical study of Mayberg and colleagues (2005), inactivation of the infralimbic cortex, the rodent correlate of the BA25 (Quirk and Beer, 2006) results in antidepressant-like behaviour in the rat modified FST. Furthermore, this behavioural response was not the result of a general increase in locomotor activity, as infusion of muscimol had no effect on this parameter over either of the two days of testing. Interestingly, activation of the region, via the $\mathrm{GABA}_{\mathrm{A}}$ receptor antagonist bicuculline, did not result in any alteration of behaviour in the FST but caused a pronounced hyperactivity. More pertinently, in $\mathrm{HAB}$ rats, inactivation of the infralimbic cortex attenuated their high inborn level of depression-related behaviour, analogous to the findings in treatment-resistant patients. These results show that it is possible to replicate findings from a clinical trial in rodents and support the use of the FST to gain greater understanding of the neurocircuitry involved in depression and antidepressant-action.

The infralimbic cortex has been implicated from preclinical studies in stressor response, working memory and also in reward-related behaviour (Cerqueira et al., 2005; Chudasama and Robbins, 2006; Coutureau and Killcross, 2003; GisquetVerrier and Delatour, 2006; Izquierdo et al., 2006). Given the role of the PFC in working and spatial memory, groups involving infusion of muscimol or bicuculline prior to the pre-test swim followed by vehicle prior to the swim test were included. These groups enabled the assessment of whether inactivation or activation during the initial stress exposure (i.e. the pre-test swim) affected the behavioural response following re-exposure. Neither acute manipulation in the current study altered behaviour in the pre-test swim exposure (Figure 1A). As previously demonstrated there was no significant difference in the pattern of active and passive behaviour between the pre-test swim and swim tests in control animals in the modified FST (Bissiere et al., 2006). However, previous studies demonstrated that pro-depressantlike effects manifested following repeated exposure to the test (Cryan et al., 2003). Further, alterations in activity of the infralimbic cortex prior to pre-test swim alone also failed to alter response in the test session (Figures $1 \mathrm{~B}$ and 1C). Therefore, the infralimbic cortex had a selective role in modulating behavioural coping responses upon re-exposure to a stressful situation. These findings are supported from the literature involving conditioned fear, which show that extinction is facilitated by increased infralimbic cortex activity and impaired by stress (Gourley et al., 2009; Quirk and Mueller, 2008). Importantly, alterations in infralimbic cortex activity, by drug manipulation or previous stress exposure, have been shown not to alter acquisition of fear suggesting a specific role of this region in modulating the recall of a stressful event (Gourley et al., 2009; Hefner et al., 2008; Quirk and Mueller, 2008). Recently, we have shown that excitotoxic lesions of the anterior cingulate cortex prior to behavioural 


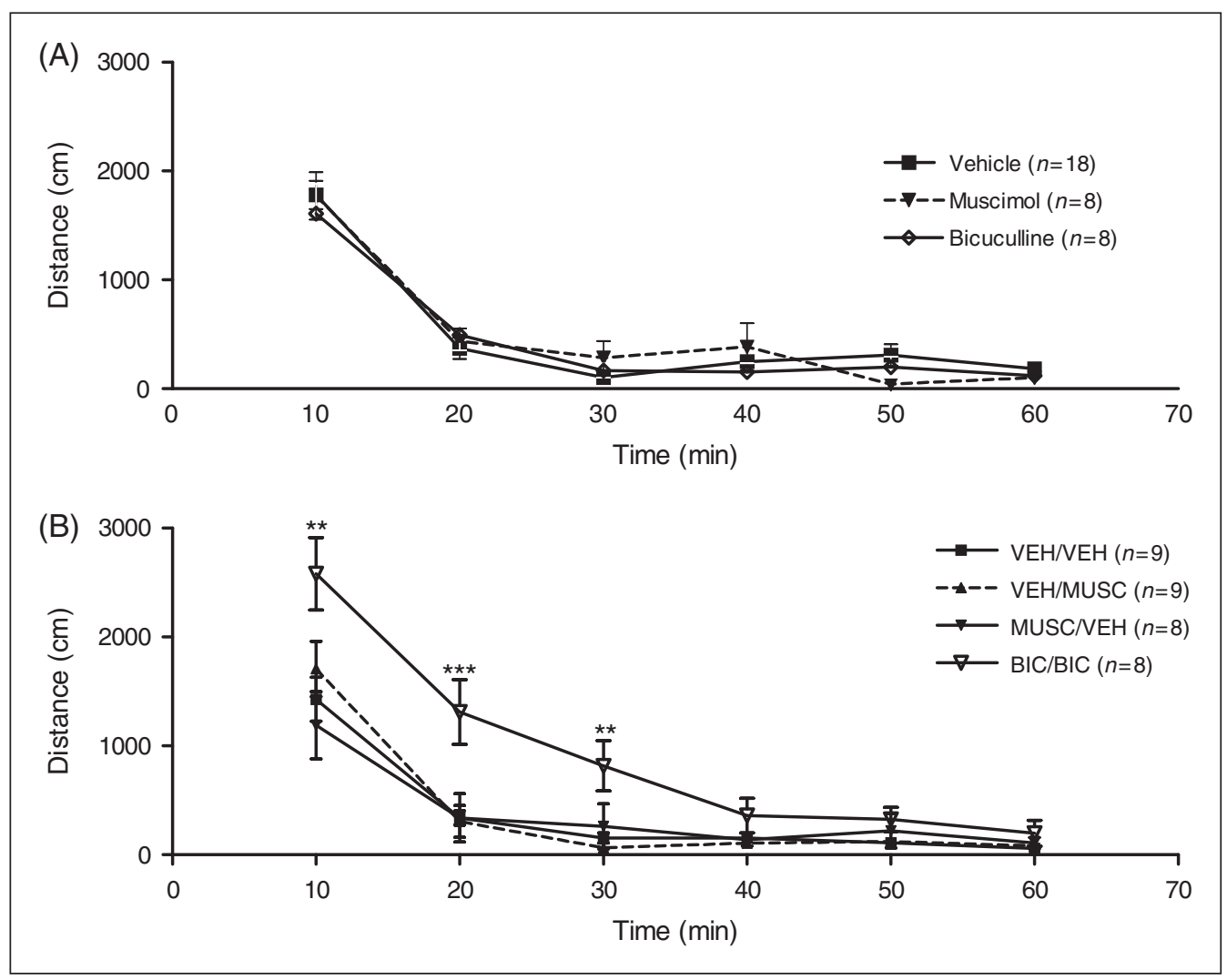

Figure 3. The effect of pharmacological inactivation/activation of the infralimbic cortex on locomotor activity. A) Neither muscimol (200 pmoles per hemisphere) nor bicuculline (50 pmoles per hemisphere) altered locomotor activity in a novel environment compared with vehicle infusion (VEH). B) Infusion of bicuculline (BIC) prior to both exposures to the locomotor test chamber resulted in a pronounced hyperactivity compared with vehicle infusion. Data represent mean \pm SEM of animals with verified bilateral cannula sites. Repeated measures ANOVA was performed followed by Fisher's post-hoc analyses where appropriate. ${ }^{* *} p<0.01$ and ${ }^{* * *} p<0.001$ compared with vehicle/vehicle group.

testing resulted in a pro-depressant-like effect (increased immobility) in the swim test but not the pre-swim test (Bissiere et al., 2006). Since the anterior cingulate cortex is spatially close $(\sim 2 \mathrm{~mm}$ posterior $)$ to the pre- and infralimbic cortices, this suggests that the antidepressant effect of muscimol in the present study is specific to the infralimbic cortex, although we cannot fully discount diffusion to the ventral prelimbic cortex. Moreover, misplaced cannulae suggested that muscimol infusion did not alter activity in either the pre-test or the swim test, although the low number of animals occludes rigorous statistical analysis.

Stress exposure also activates the hypothalamic-pituitaryadrenal (HPA) axis and lesions of the medial PFC (mPFC) have been shown to elevate HPA factors, including adrenocorticotropic hormone $(\mathrm{ACTH})$ in a manner that is dependent on the quality of the stressor (be it restraint stress, cold restraint stress, white noise exposure, IL- $1 \beta$ administration or exposure to ether fumes) and its strength (Crane et al., 2003; McDougall et al., 2004; Sullivan and Gratton, 1999). This is further exemplified by the fact that MPFC lesions increase baseline HPA function and increased ACTH concentrations and c-Fos expression in the hypothalamic paraventricular nucleus (PVN) following $20 \mathrm{~min}$ restraint stress (Sullivan and Gratton, 1999; but see Crane et al., 2003) but not following exposure to ether stress (Figueiredo et al., 2003).
Our data concur with these previous studies in indicating that $\mathrm{mPFC}$ regulates stress-responses but take them further by utilizing an animal model of stress-induced coping, which is predictive of antidepressant activity and using more regionally selective and transient disruption of neuronal activity. The hypothesis that the infralimbic cortex modulates swim stress-induced behaviours is strengthened by recent data demonstrating that repeated swim stress exposure resulted in dendritic shrinkage in the infralimbic cortex but not the prelimbic cortex and resulted in deficits in the extinction of learned fear (Izquierdo et al., 2006). The authors suggest that this retraction would lead to a decreased excitability of the infralimbic neurones, which in turn would impair its inhibitory modulation of the amygdala. Furthermore, muscimol infusion into the medial prefrontal cortex resulted in anxiolytic effects in both the elevated plus-maze and shock-probe burying tests (Shah et al., 2004). Given the substantial co-morbidity between depression and anxiety disorders (Moller, 2002) and the anxiolytic effects of chronic antidepressant administration (Borsini et al., 2002), this provides a further indication that this region plays a significant role in the pathophysiology of mood disorders. Previous studies have also demonstrated that activation of the mPFC leads to activation of c-Fos in intercalated cells of the amygdala (Berretta et al., 2005; Rosenkranz and Grace, 2002). It is believed that the infralimbic cortex 
projects to these cells, as well as the central-lateral amygdala, which are both inhibitory (Quirk and Beer, 2006; Vertes, 2004). In contrast, the more dorsal prelimbic cortex projects to the basal amygdala (Likhtik et al., 2005; Quirk and Beer, 2006). Further, human neuroimaging studies have shown that depression severity is correlated to amygdala activity (Drevets et al., 1992), which suggests that the antidepressant-like response of muscimol could be related to prevention of the stress-induced alterations in infralimbic cortex by swim exposure, resulting in increased active coping behaviour. In support of this hypothesis a recent study from Sairanen and co-workers (Sairanen et al., 2007) demonstrated that chronic antidepressant treatment selectively increased plasticity-related proteins, including a polysialylated form of nerve cell adhesion molecule, phosphorylated cyclic-AMP response element binding protein and growth-associated protein 43 in the mPFC.

Interestingly, a number of rat lines/strains implicated in depressive-related behaviour have also been shown to have dysregulation of the infralimbic cortex. Congenitally learned helpless rats, a line selectively bred for a specific trait relevant to depression, display lower metabolic activity in the infralimbic cortex at birth but this switches to hyperactivity in adulthood (Shumake et al., 2000, 2004). Moreover, destruction of astrocytes in the PFC has been shown to result in depressivelike behaviour in rodents (Banasr and Duman, 2008) and the Wistar-Kyoto strain, which exhibits high levels of anxiety and depression-related behaviour, has been shown to exhibit a reduced number of glial cells in the infralimbic cortex (Gosselin et al., 2009). The present study examined HAB rats, which have a high inborn level of depression-like behaviour and have previously been shown to have altered neuronal activity in the infralimbic cortex in response to anxiogenic or anxiolytic administration (Kalisch et al., 2004; Singewald, 2006). Polymorphisms in the promoter region of the arginine vasopressin (AVP) gene have been shown to result in high and low expression and intrahypothalamic release of AVP in HAB and LAB rats, respectively (Landgraf et al., 2007; Murgatroyd et al., 2004). Moreover, these differences related to the AVP system have been causally linked to the difference in inborn anxiety between the lines (see Landgraf et al., 2007 for a review). Previously, chronic treatment with paroxetine over 8 weeks (Keck et al., 2003) or direct infusion of an AVP-receptor antagonist into the PVN (Wigger et al., 2004) have been shown to decrease the high depression-related behaviour in HAB rats. However, no other treatment, including chronic infusion of oxytocin, a neuropeptide related to AVP with anxiolytic and partly anti-depressive effects (Slattery and Neumann, 2010), has been shown to alter depression-related behaviour in these rats to date. Therefore, the fact that transient inactivation of the infralimbic cortex resulted in an attenuation of this high depression-related behaviour suggests a strong role of the region in the behavioural phenotype of HAB rats (Figure 2). This may possibly be mediated via infralimbic control of the HPA axis response to stress exposure, which is elevated in HAB rats (Landgraf et al., 2007; Murgatroyd et al., 2004). However, further experiments are required to verify this. Importantly, the results in $\mathrm{HAB}$ rats strengthen the possibility that altered activity of infralimbic cortex in rodent or the BA25 in humans results in the increased likelihood of depressionrelated behaviour.
The antidepressant-like effect of inactivation of the infralimbic cortex was reflected by a selective increase in climbing behaviour in both Sprague-Dawley and HAB rats, which is known to be mediated by increased catecholamine transmission (Cryan et al., 2002; Slattery et al., 2005). Interestingly, chronic stress has been shown to cause a down-regulation of dopamine D1 receptors in the infralimbic cortex (Goldwater et al., 2009) and early-life stress has been shown to alter noradrenergic-, but not serotonergic-, axon density in this region (Kuramochi and Nakamura, 2009). Therefore, it is possible that acute stress also predominantly affects dopaminergic and noradrenergic transmission, and inactivation of the region restores the balance.

Although originally designed as a screening tool for antidepressants (Porsolt et al., 1977), FST behaviour has been shown to be regulated by various pharmacological and nonpharmacological factors that also alter depression-related behaviours in humans. These are as diverse as genetic predisposition, prior exposure to stress, diabetes, alterations in sleep architecture, and drug-withdrawal-induced anhedonia (Cryan and Holmes, 2005; Cryan et al., 2003, 2005). Although the FST has been in use for over three decades, the brain regions and neural circuitry underlying behaviours in this paradigm remain rather elusive. Previous studies have described a role of different regions such as the lateral septum (Price et al., 2002), the amygdala (Ebner et al., 2002), the bed nucleus of stria terminalis (Schulz and Canbeyli, 2000) and the rostral anterior cingulate cortex (Bissiere et al., 2006). Moreover, recent data correlates stress-sensitive hippocampal activity with immobility in the FST (Airan et al., 2007). Our present data demonstrate that the modulation of infralimbic cortex activity, the rodent correlate of BA 25, alters FST behaviour in an analogous fashion to that shown by DBS in humans. This further strengthens the face validity of the FST and suggests that it may be a valuable tool to probe the underlying mechanisms of disorders of stress-induced coping, such as depression.

\section{Acknowledgements}

This work was supported by National Institutes of Mental Health/ National Institute on Drug Abuse Grant U01 MH69062. The authors would like to thank Hugo Buerki, Stefan Imobersteg and Rodrigue Maloumby for technical assistance, and Dr Daniela Beiderbeck for supporting the breeding of HAB rats.

\section{References}

Airan RD, Meltzer LA, Roy M, Gong Y, Chen H and Deisseroth K (2007) High-speed imaging reveals neurophysiological links to behavior in an animal model of depression. Science 317: 819-823.

Amat J, Baratta MV, Paul E, Bland ST, Watkins LR and Maier SF (2005) Medial prefrontal cortex determines how stressor controllability affects behavior and dorsal raphe nucleus. Nat Neurosci 8: 365-371.

Anisman H and Zacharko RM (1990) Multiple neurochemical and behavioral consequences of stressors: implications for depression. Pharmacol Ther 46: 119-136.

Banasr M and Duman RS (2008) Glial loss in the prefrontal cortex is sufficient to induce depressive-like behaviors. Biol Psychiatry 64: 863-870. 
Barbas H, Saha S, Rempel-Clower N and Ghashghaei T (2003) Serial pathways from primate prefrontal cortex to autonomic areas may influence emotional expression. BMC Neurosci 4: 25.

Berretta S, Pantazopoulos H, Caldera M, Pantazopoulos P and Pare D (2005) Infralimbic cortex activation increases c-Fos expression in intercalated neurons of the amygdala. Neuroscience 132: 943-953.

Bissiere S, McAllister KH, Olpe HR and Cryan JF (2006) The rostral anterior cingulate cortex modulates depression but not anxietyrelated behaviour in the rat. Behav Brain Res 175: 195-199.

Borsini F, Lecci A, Sessarego A, Frassine R and Meli A (1989) Discovery of antidepressant activity by forced swimming test may depend on pre-exposure of rats to a stressful situation. Psychopharmacology (Berl) 97: 183-188.

Borsini F, Podhorna J and Marazziti D (2002) Do animal models of anxiety predict anxiolytic-like effects of antidepressants? Psychopharmacology (Berl) 163: 121-141.

Bremner JD (2002) Structural changes in the brain in depression and relationship to symptom recurrence. CNS Spectr 7: 129-130, 135-139.

Cerqueira JJ, Pego JM, Taipa R, Bessa JM, Almeida OF and Sousa $\mathrm{N}$ (2005) Morphological correlates of corticosteroid-induced changes in prefrontal cortex-dependent behaviors. J Neurosci 25: 7792-7800.

Chudasama Y and Robbins TW (2006) Functions of frontostriatal systems in cognition: comparative neuropsychopharmacological studies in rats, monkeys and humans. Biol Psychol 73: 19-38.

Corcoran KA, Desmond TJ, Frey KA and Maren S (2005) Hippocampal inactivation disrupts the acquisition and contextual encoding of fear extinction. $J$ Neurosci 25: 8978-8987.

Coutureau E and Killcross S (2003) Inactivation of the infralimbic prefrontal cortex reinstates goal-directed responding in overtrained rats. Behav Brain Res 146: 167-174.

Crane JW, Ebner K and Day TA (2003) Medial prefrontal cortex suppression of the hypothalamic-pituitary-adrenal axis response to a physical stressor, systemic delivery of interleukin-1beta. Eur J Neurosci 17: 1473-1481.

Cryan JF and Holmes A (2005) The ascent of mouse: advances in modelling human depression and anxiety. Nat Rev Drug Discov 4: 775-790.

Cryan JF, Hoyer D and Markou A (2003) Withdrawal from chronic amphetamine induces depressive-like behavioral effects in rodents. Biol Psychiatry 54: 49-58.

Cryan JF and Lucki I (2000a) 5-HT4 receptors do not mediate the antidepressant-like behavioral effects of fluoxetine in a modified forced swim test. Eur J Pharmacol 409: 295-299.

Cryan JF and Lucki I (2000b) Antidepressant-like behavioral effects mediated by 5 -Hydroxytryptamine(2C) receptors. J Pharmacol Exp Ther 295: 1120-1126.

Cryan JF, Markou A and Lucki I (2002) Assessing antidepressant activity in rodents: recent developments and future needs. Trends Pharmacol Sci 23: 238-245.

Cryan JF and Slattery DA (2007) Animal models of mood disorders: Recent developments. Curr Opin Psychiatry 20: 1-7.

Cryan JF, Valentino RJ and Lucki I (2005) Assessing substrates underlying the behavioral effects of antidepressants using the modified rat forced swimming test. Neurosci Biobehav Rev 29: 547-569.

Davidson RJ, Pizzagalli D, Nitschke JB and Putnam K (2002) Depression: perspectives from affective neuroscience. Annu Rev Psychol 53: 545-574.

Dias-Ferreira E, Sousa JC, Melo I, et al. (2009) Chronic stress causes frontostriatal reorganization and affects decision-making. Science 325: 621-625.

Dickinson-Anson H and McGaugh JL (1997) Bicuculline administered into the amygdala after training blocks benzodiazepineinduced amnesia. Brain Res 752: 197-202.
Dougherty DD, Weiss AP, Cosgrove GR, et al. (2003) Cerebral metabolic correlates as potential predictors of response to anterior cingulotomy for treatment of major depression. J Neurosurg 99: $1010-1017$.

Drevets WC (2001) Neuroimaging and neuropathological studies of depression: implications for the cognitive-emotional features of mood disorders. Curr Opin Neurobiol 11: 240-249.

Drevets WC, Videen TO, Price JL, Preskorn SH, Carmichael ST and Raichle ME (1992) A functional anatomical study of unipolar depression. J Neurosci 12: 3628-3641.

Ebner K, Wotjak CT, Landgraf R and Engelmann M (2002) Forced swimming triggers vasopressin release within the amygdala to modulate stress-coping strategies in rats. Eur $J$ Neurosci 15: 384-388.

Figueiredo HF, Bruestle A, Bodie B, Dolgas CM and Herman JP (2003) The medial prefrontal cortex differentially regulates stressinduced c-fos expression in the forebrain depending on type of stressor. Eur J Neurosci 18: 2357-2364.

Gisquet-Verrier P and Delatour B (2006) The role of the rat prelimbic/infralimbic cortex in working memory: not involved in the short-term maintenance but in monitoring and processing functions. Neuroscience 141: 585-596.

Goldapple K, Segal Z, Garson C, et al. (2004) Modulation of cortical-limbic pathways in major depression: treatment-specific effects of cognitive behavior therapy. Arch Gen Psychiatry 61: 34-41.

Goldwater DS, Pavlides C, Hunter RG, et al. (2009) Structural and functional alterations to rat medial prefrontal cortex following chronic restraint stress and recovery. Neuroscience 164: 798-808.

Gosselin RD, Gibney S, O'Malley D, Dinan TG and Cryan JF (2009) Region specific decrease in glial fibrillary acidic protein immunoreactivity in the brain of a rat model of depression. Neuroscience 159: 915-925.

Gourley SL, Kedves AT, Olausson P and Taylor JR (2009) A history of corticosterone exposure regulates fear extinction and cortical NR2B, GluR2/3, and BDNF. Neuropsychopharmacology 34: 707-716.

Hains AB, Vu MA, Maciejewski PK, van Dyck CH, Gottron M and Arnsten AF (2009) Inhibition of protein kinase C signaling protects prefrontal cortex dendritic spines and cognition from the effects of chronic stress. Proc Natl Acad Sci USA 106: 17957-17962.

Hefner K, Whittle N, Juhasz J, et al. (2008) Impaired fear extinction learning and cortico-amygdala circuit abnormalities in a common genetic mouse strain. $J$ Neurosci 28: 8074-8085.

Izquierdo A, Wellman CL and Holmes A (2006) Brief uncontrollable stress causes dendritic retraction in infralimbic cortex and resistance to fear extinction in mice. $J$ Neurosci 26: 5733-5738.

Jahn AL, Fox AS, Abercrombie HC, et al. (2009) Subgenual Prefrontal cortex activity predicts individual differences in hypothalamic-pituitary-adrenal activity across different contexts. Biol Psychiatry 67: 175-181.

Kalisch R, Salome N, Platzer S, et al. (2004) High trait anxiety and hyporeactivity to stress of the dorsomedial prefrontal cortex: a combined phMRI and Fos study in rats. Neuroimage 23: 382-391.

Keck ME, Welt T, Muller MB, et al. (2003) Reduction of hypothalamic vasopressinergic hyperdrive contributes to clinically relevant behavioral and neuroendocrine effects of chronic paroxetine treatment in a psychopathological rat model. Neuropsychopharmacology 28: 235-243.

Kessler RC (1997) The effects of stressful life events on depression. Annu Rev Psychol 48: 191-214.

Kuramochi M and Nakamura S (2009) Effects of postnatal isolation rearing and antidepressant treatment on the density of serotonergic and noradrenergic axons and depressive behavior in rats. Neuroscience 163: 448-455. 
Landgraf R, Kessler MS, Bunck M, et al. (2007) Candidate genes of anxiety-related behavior in $\mathrm{HAB} / \mathrm{LAB}$ rats and mice: focus on vasopressin and glyoxalase-I. Neurosci Biobehav Rev 31: 89-102.

Landgraf R and Wigger A (2002) High vs low anxiety-related behavior rats: an animal model of extremes in trait anxiety. Behav Genet 32: $301-314$.

Likhtik E, Pelletier JG, Paz R and Pare D (2005) Prefrontal control of the amygdala. $J$ Neurosci 25: 7429-7437.

Maren S, Yap SA and Goosens KA (2001) The amygdala is essential for the development of neuronal plasticity in the medial geniculate nucleus during auditory fear conditioning in rats. J Neurosci 21: RC135.

Mayberg HS, Liotti M, Brannan SK, et al. (1999) Reciprocal limbiccortical function and negative mood: converging PET findings in depression and normal sadness. Am J Psychiatry 156: 675-682.

Mayberg HS, Lozano AM, Voon V, et al. (2005) Deep brain stimulation for treatment-resistant depression. Neuron 45: 651-660.

McDougall SJ, Widdop RE and Lawrence AJ (2004) Medial prefrontal cortical integration of psychological stress in rats. Eur $J$ Neurosci 20: 2430-2440.

Moller HJ (2002) Anxiety associated with comorbid depression. $J$ Clin Psychiatry 63(Suppl 14): 22-26.

Murgatroyd C, Wigger A, Frank E, et al. (2004) Impaired repression at a vasopressin promoter polymorphism underlies overexpression of vasopressin in a rat model of trait anxiety. $J$ Neurosci 24: $7762-7770$.

Neumann ID (2009) The advantage of living social: brain neuropeptides mediate the beneficial consequences of sex and motherhood. Front Neuroendocrinol 30: 483-496.

Ongur D and Price JL (2000) The organization of networks within the orbital and medial prefrontal cortex of rats, monkeys and humans. Cereb Cortex 10: 206-219.

Paxinos G and Watson C (1986) The Rat Brain in Sterotaxic Coordinates. New York: Academic Press.

Perez-Cruz C, Simon M, Flugge G, Fuchs E and Czeh B (2009) Diurnal rhythm and stress regulate dendritic architecture and spine density of pyramidal neurons in the rat infralimbic cortex. Behav Brain Res 205: 406-413.

Petit-Demouliere B, Chenu F and Bourin M (2005) Forced swimming test in mice: a review of antidepressant activity. Psychopharmacology (Berl) 177: 245-255.

Porsolt RD, Le Pichon M and Jalfre M (1977) Depression: a new animal model sensitive to antidepressant treatments. Nature 266: 730-732.

Pothuizen HH, Jongen-Relo AL and Feldon J (2005) The effects of temporary inactivation of the core and the shell subregions of the nucleus accumbens on prepulse inhibition of the acoustic startle reflex and activity in rats. Neuropsychopharmacology 30: 683-696.

Price ML, Kirby LG, Valentino RJ and Lucki I (2002) Evidence for corticotropin-releasing factor regulation of serotonin in the lateral septum during acute swim stress: adaptation produced by repeated swimming. Psychopharmacology (Berl) 162: 406-414.

Quirk GJ and Beer JS (2006) Prefrontal involvement in the regulation of emotion: convergence of rat and human studies. Curr Opin Neurobiol 16: 723-727.
Quirk GJ and Mueller D (2008) Neural mechanisms of extinction learning and retrieval. Neuropsychopharmacology 33: 56-72.

Rosenkranz JA and Grace AA (2002) Cellular mechanisms of infralimbic and prelimbic prefrontal cortical inhibition and dopaminergic modulation of basolateral amygdala neurons in vivo. $J$ Neurosci 22: 324-337.

Sairanen M, O'Leary OF, Knuuttila JE and Castren E (2007) Chronic antidepressant treatment selectively increases expression of plasticity-related proteins in the hippocampus and medial prefrontal cortex of the rat. Neuroscience 144: 368-374.

Schulz D and Canbeyli RS (2000) Lesion of the bed nucleus of the stria terminalis enhances learned despair. Brain Res Bull 52: $83-87$.

Seminowicz DA, Mayberg HS, McIntosh AR, et al. (2004) Limbicfrontal circuitry in major depression: a path modeling metanalysis. Neuroimage 22: 409-418.

Shah AA, Sjovold T and Treit D (2004) Inactivation of the medial prefrontal cortex with the GABAA receptor agonist muscimol increases open-arm activity in the elevated plus-maze and attenuates shock-probe burying in rats. Brain Res 1028: 112-115.

Sheline YI, Mittler BL and Mintun MA (2002) The hippocampus and depression. Eur Psychiatry 17( Suppl 3): 300-305.

Shumake J, Conejo-Jimenez N, Gonzalez-Pardo H and GonzalezLima F (2004) Brain differences in newborn rats predisposed to helpless and depressive behavior. Brain Res 1030: 267-276.

Shumake J, Poremba A, Edwards E and Gonzalez-Lima F (2000) Congenital helpless rats as a genetic model for cortex metabolism in depression. Neuroreport 11: 3793-3798.

Singewald N (2006) Altered brain activity processing in high-anxiety rodents revealed by challenge paradigms and functional mapping. Neurosci Biobehav Rev 31: 18-40.

Slattery DA, Markou A, Froestl W and Cryan JF (2005) The GABAB receptor-positive modulator GS39783 and the GABAB receptor agonist baclofen attenuate the reward-facilitating effects of cocaine: intracranial self-stimulation studies in the rat. Neuropsychopharmacology 30: 2065-2072.

Slattery DA and Neumann ID (2010) Chronic icv oxytocin attenuates the pathological high anxiety state of selectively bred Wistar rats. Neuropharmacology 58: 56-61.

Sullivan RM and Gratton A (1999) Lateralized effects of medial prefrontal cortex lesions on neuroendocrine and autonomic stress responses in rats. J Neurosci 19: 2834-2840.

Sullivan PF, Neale MC and Kendler KS (2000) Genetic epidemiology of major depression: review and meta-analysis. Am J Psychiatry 157: $1552-1562$.

Uylings HB, Groenewegen HJ and Kolb B (2003) Do rats have a prefrontal cortex? Behav Brain Res 146: 3-17.

Vertes RP (2004) Differential projections of the infralimbic and prelimbic cortex in the rat. Synapse 51: 32-58.

Wigger A, Sanchez MM, Mathys KC, et al. (2004) Alterations in central neuropeptide expression, release, and receptor binding in rats bred for high anxiety: critical role of vasopressin. Neuropsychopharmacology 29: 1-14.

Zhang WN, Bast T and Feldon J (2002) Prepulse inhibition in rats with temporary inhibition/inactivation of ventral or dorsal hippocampus. Pharmacol Biochem Behav 73: 929-940. 